\title{
Exploring the Role of Oral Reading Fluency (ORF) in Reading Instruction: A Classroom- based Study with Engineering Students
}

\author{
Remya Rebecca Prakash* and Abjy Kurian ${ }^{\dagger}$
}

\begin{abstract}
Teaching reading is a complicated task as reading entails the simultaneous interaction of various component processes. Learning to read fluently is a crucial part of reading acquisition. Despite research conducted in L1 contexts regarding reading fluency, this area has been relatively unexplored in L2 contexts. In reading fluency research, Oral Reading Fluency (ORF) is an important construct which has been closely associated with the reading comprehension of young learners in L1 contexts. This paper aims to investigate the nature of the relationship between ORF and reading comprehension in ESL learners. If ORF proves to be significantly related to reading comprehension, it would be a valuable addition to reading instruction for ESL learners.
\end{abstract}

Keywords: Oral Reading Fluency, Reading Comprehension, ESL Learners

\section{Introduction}

Reading is a complex process which involves the translation of a text into the spoken language with the aim of deriving meaning

\footnotetext{
English and Foreign Languages University, Hyderabad, India; remyaprksh@gmail.com

$\dagger$ National Institute of Technology, Tiruchirapalli, India; abjy16.08@gmail.com
} 
from it (Gibson, 1965; Perfetti, 1999; Alderson, 2000). When a text is read, a number of component processes are concurrently coordinated and reading acquisition requires the mastery of these processes. In first language contexts, reading fluency has been recognised as a key element of reading acquisition (National Reading Panel, 2000; Kuhn \& Stahl, 2003). This is evidenced in the initial stages of learning to read aloud: when young learners read aloud, oral comprehension skills are used to understand a text by listening to themselves. Therefore, the manner in which the text is read aloud is important. When a text is read aloud accurately, at a conversational pace, with appropriate phrasing and expression, the text is considered to be read fluently, and the construct that measures it is called Oral Reading Fluency (ORF). This multidimensional construct has been closely associated with reading comprehension in L1 contexts. However, comprehensive research associated with ORF and its three elements-accuracy, automaticity, and prosody-in ESL populations are limited. Although reading fluency is considered as an issue pertaining to young learners, this study explores the role of oral reading fluency in instructional practice in higher education. As the preferred modality of reading in higher educational contexts is silent reading, the relationship between oral reading comprehension and silent reading comprehension is also explored.

ORF is a construct that can be observed when a meaningful text is read aloud. It has been defined as "the accurate reading of connected text at a conversational rate with appropriate prosody or expression" (Hudson et al., 2005, p. 702). This definition highlights the multidimensional nature of ORF where "one dimension stresses the importance of accuracy in word decoding, a second dimension focuses on quick and automatic recognition of words in connected text, and a third dimension stresses expressive and meaningful interpretation of text" (Rasinski, 2004, p. 6). Apart from being central to defining ORF, these elements are related to each other, equally significant to fluent reading (Rasinski, 2004; Grabe, 2009) and are themselves cognitively complex. Accuracy is not merely the accurate decoding of words, but it is closely related to word recognition and retrieval, while automaticity goes beyond speed reading of a text and signifies unconscious processing of text. Similarly, prosody indicates "the recognition of prosodic phrasing 
and contours of the text" (Rasinski, 2004, p.6) which are closely linked to syntactic and semantic processes. Prosody is considered to enable major informational units (like phrases) to be cognitively bracketed for a later semantic analysis to be carried out (Benjamin and Schwanenflugel, 2010). Therefore, it is not only accuracy and automaticity that reduce the cognitive load of the reader, but prosody also plays a role in enhancing the process of comprehension.

A majority of the studies based on ORF have dealt with native speakers of English. However, research on ORF in ESL learners is very limited, despite the rapidly increasing number of non-native speakers. It is important to understand how ORF develops in ESL learners, and whether the research findings for native speakers are similar for ESL learners as well. As the development of ORF in ESL learners has not yet been verified, it is necessary to examine the nature of the relationship between ORF and reading comprehension in older learners as well. The current study aimed to determine the role of ORF in the reading instruction of engineering students, across ability groups. The objectives of the study were: (a) to examine the relationship between ORF and reading comprehension in these learners, and (b) to examine the relationship between oral reading comprehension and silent reading comprehension in these learners.

\subsection{Profile of the Sample}

The participants of the study were 27 first-year engineering students from the National Institute of Technology, Tiruchirapalli. The sample consisted of a higher number of males to females (21 boys, 6 girls). While a majority of the sample were from India $(n=24)$, there were some students from Bangladesh $(n=2)$ and Sri Lanka $(n=1)$. The medium of instruction in school for the majority of the sample was English (88\%), though some learners were from Hindi (8\%) and Bangla (4\%) medium. The sample was divided into three groups - above average, average, and below average-based on their performances in a language test that was conducted before the commencement of this study and their language teacher's sense of plausibility (Kumaravadivelu, 1994). There were nine learners in 
each group, and these groups were, however, not made evident to the learners.

\section{Methodology}

The sample were administered an oral reading assessment and a silent reading assessment. Two texts of comparable length and difficulty were chosen for these assessments. The text used for the oral reading assessment was The Touchstone (word count: 531) (Norris, 2012), and the text for the silent reading assessment was Ctrl-Z (word count: 603) (Norris, 2012).

\section{Assessment of Oral Reading}

For the administration of the oral reading assessment, the examiner (Abjy Kurian) met the learners individually. The learners were asked to read the text aloud, and the recordings of the oral readings were assessed in terms of accuracy, automaticity, and prosody. The oral reading of each learner was transcribed and analysed. The scoring and analysis of these elements of ORF were done as follows:

\subsection{Accuracy: Miscue analysis}

The miscues made by the learners in their oral reading were noted and categorised according to the type of miscue. Six types of miscues were identified: correction, insertion, omission, repetition, reversal, and substitution (Argyle, 1989). The accuracy score was calculated as the percentage of words read correctly by a learner when reading a text. For a learner to read in an independent manner, it is recommended that they read at $97 \%$ accuracy (Hasbrouck \&Tindal, 1992). The type of miscues was further analysed on the basis of meaning-change in order to ascertain whether the miscues were detrimental to comprehension or not. To this effect, miscues of Substitution and Omission were analysed in relation to the reading comprehension questions. When a learner substitutes or omits a word when reading aloud, there is a chance that the learner does not correctly understand what is written in the text, thereby causing a lapse in comprehension. Therefore, each learner's transcripts were analysed to check the type of miscues 
that occurred at specific points in the text on which the comprehension questions were based.

\subsection{Automaticity: Number of Words Read Per Minute}

The automaticity score was calculated by dividing the total number of words in the text by the time taken by the learner to read the text. From the assessment of fluent reading, 250-300 words per minute has been generally accepted as the rate at which fluent readers read (Carver, 1997; Grabe, 2009).

\subsection{Prosody: Multidimensional Fluency Scoring Guide (Rasinski et al., 2009)}

This fluency scoring guide assessed the oral reading of a text in terms of three aspects: Phrasing and Expression, Accuracy and Smoothness, and Pacing. This scale provides analytic scoring using a Likert scale of 1-4 such that a learner gets at least a minimum score of 1 and a maximum score of 4 for each criterion. Scores above 8 indicate good progress while scores below 8 show that the student is struggling (Rasinski et al., 2009). Abjy Kurian and Remya Prakash assessed the prosody of the learners from the audio recordings. The average of the scores of the two raters was calculated as the final prosody score.

After the learners read the text aloud, they were orally administered a comprehension test. The oral responses of each learner were noted separately. The test focussed on assessing the readers in terms of the following reading sub-skills:

1. Understanding explicitly stated information and scanning the text to locate specifically required information

2. Identifying main points or important information in a text

3. Deducing the meaning of unfamiliar lexical terms from context

4. Inferring information when not explicitly stated

\section{Assessment of Silent Reading}

Similar to the assessment of oral reading, the researcher met with each learner individually for the assessment of silent reading as 
well. The learners were asked to read the text silently and indicate to the researcher when they had finished reading the text. The time taken by the learners to finish reading the text was noted. To calculate the silent reading rate, the total number of words in the text was divided by the time taken by the learners to complete reading the text. It is necessary to note at this point that rate in silent reading may not accurately measure the time taken for the learner to read the text. As this research did not use any eye tracking equipment (like SMI Eyelink I or SR Research Eyelink 1000), it is not possible to measure the exact time that the learner used to read the text once; it is likely that the learner re-read a certain sentence/paragraph multiple times for better understanding (which is commonly done when one reads a text silently). Therefore, here the learners are asked to indicate when they have finished reading as a means to ensure that the learner does not take too much time to read the text. After the learner completed reading the text, a reading comprehension test based on the reading sub-skills (mentioned before) was orally administered. The oral responses of each learner were noted separately.

\section{Results: Analysis and Interpretation}

\subsection{Oral Reading Assessment}

The mean scores and standard deviations for the elements of ORF and reading comprehension for the oral reading assessment of each ability group are given in the table below. Here, Group 2A denotes the above average students, Group A denotes the average students, and Group B, the below average students.

Table 1: Results of ORF elements and Reading Comprehension

\begin{tabular}{lrrrrrrrr}
\hline & \multicolumn{2}{c}{ Accuracy } & \multicolumn{2}{c}{ Automaticity } & \multicolumn{2}{c}{ Prosody } & \multicolumn{2}{c}{ RC } \\
\cline { 2 - 9 } Group 2A & Average & SD & Average & SD & Average & SD & Average & SD \\
\cline { 2 - 9 } Group A & 98.97 & 0.44 & 212.52 & 16.55 & 10 & 1.62 & 5.15 & 1.17 \\
Group B & 97.76 & 1.62 & 167.87 & 37.43 & 6.94 & 1.55 & 4.11 & 1.67 \\
\hline
\end{tabular}

From the above table, it can be seen that all the groups have an average percentage score of accuracy that is above $97 \%$, which, as mentioned before, presumes that the learners are able to read in an independent manner. This suggests that they do not produce 
enough miscues that may hinder their comprehension. However, none of the groups has average automaticity scores within the range of fluent reading, i.e. 250-300 wpm. This may imply that the learners have not reached a level of automaticity in which the component processes of reading (such as word recognition and syntactic parsing) take place without any conscious effort. In terms of prosody, only Group 2A has an average score of 8 or above (indicating good progress in prosodic reading). The prosody results of Groups A and B suggest that they have not been able to appropriately process the text, syntactically or semantically. The average reading comprehension scores of the three groups show that none of the groups has performed exceptionally well. The reading comprehension scores also indicate the existence of a hierarchy between the groups (with Group 2A performing better than Groups A and B), as seen in the average scores of the ORF elements as well.

The types of miscues of the learners were also analysed at the different comprehension points in the text. Unnecessary, prolonged Pauses and two types of miscues, Substitution and Omission, were counted at the points in the text on which the comprehension questions were based.

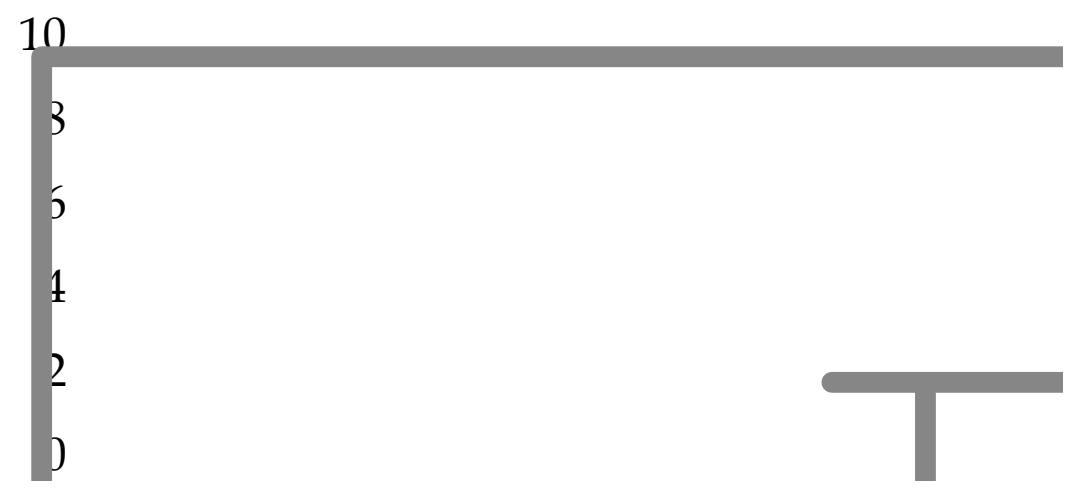

Graph 1: Type of Miscues at Comprehension Testing Points

From the graph above, it can be seen that Groups A and B have more Substitution miscues than Omission and Pause miscues. In Group B, there were 34 incorrect answers, of which 8 Substitution miscues, 1 Omission miscue and 2 Pauses were noted at the 
comprehension testing points in the text. For Group A, the learners answered 32 questions incorrectly, while there were 9 Substitution miscues and 2 Pauses at the comprehension testing points. Thus it can be understood that at crucial points in the text where comprehension is essential, Groups A and B have produced Substitution and Omission miscues which may have affected their comprehension. With regard to Group 2A, the learners answered 24 questions incorrectly, but there were no miscues at the comprehension testing points. This result points to factors other than miscues which could be hindering their comprehension - they could have issues with their semantic processing, syntactic processing, or even higher order processing skills such as establishing a propositional meaning or inferencing (Weir et al., 2009). A closer look at the questions which were answered incorrectly shows that Group 2A had issues with the inferential questions specifically, while Groups A and B had problems with all the reading sub-skills that were assessed. From this analysis, it is clear that the average accuracy percentage of the groups which was discussed before was perceptually deceptive - they appear to be reading in an independent manner but this closer analysis reveals the lack of comprehension despite over $97 \%$ accuracy.

In order to closely examine the nature of the relationship between the elements of ORF (accuracy, automaticity, and prosody) and reading comprehension, the Pearson product-moment correlation coefficients $(\mathrm{r})$ of these elements and reading comprehension of the whole sample were computed (as indicated in the table given below).

Table 2: Correlation between ORF elements and Reading Comprehension

\begin{tabular}{crrr}
\hline Correlation & Accuracy & Automaticity & Prosody \\
Reading Comprehension & 0.228 & $0.414^{*}$ & 0.314 \\
\hline
\end{tabular}

${ }^{*}$ Correlation is significant at the 0.05 level

It can be seen that there are weak positive relationships between all the elements of ORF and reading comprehension. However, only the correlation between automaticity and reading comprehension shows a significant relationship at the 0.05 level. The lack of significant correlation with accuracy could be due to the vagaries 
that were noticed earlier-high accuracy levels did not ensure comprehension. In the case of prosody, this result could be caused by the learners' transition from oral reading to silent reading as the preferred modality of reading. Once a reader successfully transitions to the silent reading modality, he/she would be able to process the semantic and syntactic structures of the text without orally producing prosodic phrasing and contours. The Pearson product-moment correlation coefficients $(r)$ of these elements and reading comprehension of the ability groups were also computed (as indicated in the table given below).

Table 3: Correlations between ORF elements and Reading Comprehension (Ability Groups)

\begin{tabular}{llll}
\hline Correlation with RC & \multicolumn{1}{c}{ Accuracy } & Automaticity & \multicolumn{1}{c}{ Prosody } \\
\hline Group 2A & -0.24 & $0.77^{*}$ & $-0.69^{*}$ \\
Group A & -0.21 & 0.22 & 0.22 \\
Group B & 0.56 & -0.01 & 0.45 \\
\hline
\end{tabular}

*Correlation is significant at the 0.05 level

In the correlations of accuracy and reading comprehension, it can be seen that there are weak negative relationships for Group 2A and $\mathrm{A}(\mathrm{r}=-0.24,-0.21)$. This would imply that as accuracy increases, comprehension decreases. This inverse relationship could be a result of a phenomenon called 'word calling' - 'word callers' are learners who can pronounce words accurately without any understanding of what they are reading. This could be a result of the over-emphasis given to accuracy in reading instruction at the school level. However, in Group B, a moderate positive relationship is evident $(\mathrm{r}=0.56)$. This could be attributed to decoding or word recognition issues, which causes them to pronounce words inaccurately, thereby obstructing their comprehension.

In terms of automaticity, the relationship with reading comprehension varies between the groups. Group 2A has a strong, statistically significant correlation $(\mathrm{r}=0.77)$. As the reading pace of these learners increase, their comprehension increases because the component processes of reading are automatised, allowing them to concentrate on carrying out the higher level processes of reading, which are associated with comprehension. Both Groups A and B exhibit weak correlations $(\mathrm{r}=0.22,-0.01)$ indicating the near absence of a relationship between automaticity and reading 
comprehension. Both these groups exhibited low average scores of automaticity.

With regard to prosody, Group 2A shows a strong, negative, statistically significant correlation $(\mathrm{r}=-0.69)$ with reading comprehension. This implies that as the prosody scores decrease, the reading comprehension scores increase. As the reading comprehension scores of this group are higher than the other two groups, this correlation would mean that they are not dependent on prosodic reading for syntactic and semantic processing; they may have already successfully transitioned to the silent reading modality. Group A shows a weak positive relationship $(\mathrm{r}=0.22)$ between prosody and reading comprehension, while Group B exhibits a moderate positive relationship $(r=0.45)$. Therefore, reading comprehension scores increase as prosody scores increase for learners in Groups A and B, but it is more clearly seen in Group B. Learners in Group B may be more reliant on orally pausing at appropriate places and reading with expression in order to process the text, while some Group A learners may be more accustomed to reading silently and processing the text without explicit, prosodic reading.

\section{Silent Reading Assessment}

The comprehension scores of the silent reading assessment were compared to the oral reading comprehension scores. As seen in the graph below, the learners of each group had higher silent reading comprehension scores than oral reading comprehension scores.

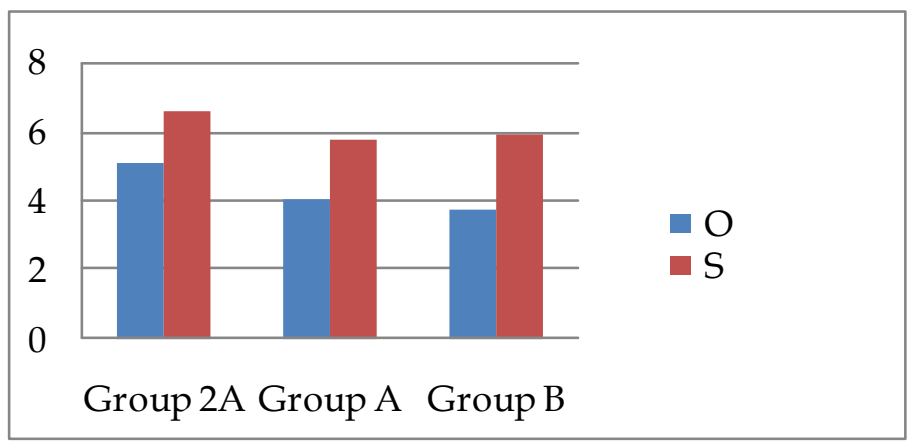

Graph 2: Oral and Silent Reading Comprehension Scores: Group Performances 
The higher silent reading comprehension scores in all the ability groups could be because silent reading is the usual modality of reading for these learners. On the other hand, after the assessments, $56 \%$ of the learners had informed the examiner that they preferred oral reading, while only $44 \%$ of the learners preferred silent reading. The reason they cited for their preference of oral reading was that it helped them to concentrate on what they were reading.

\section{Implications and Limitations of the Study}

The analysis of the results shows us that though the overall relationship between the elements of ORF and reading comprehension is weak, the correlations for the ability groups vary. The above average learners (Group 2A) show characteristics of having successfully transitioned to the silent reading modality, though they experience problems with higher order reading skills, one of them being inferencing. The average learners (Group A) are in a mixed state-some learners seem to be similar to the above average learners, while others still have issues with basic aspects of accuracy, automaticity, and prosody. The below average learners (Group B) appear to have issues with the three elements of ORF, which may be inhibiting their comprehension.

From this study, it can be suggested that incorporating ORF training as part of remedial instruction for engineering students would prove beneficial for learners who have not yet mastered the elements of ORF. ORF training would imply that equal importance is given to each element of ORF, unlike the fluency training they have received in school, which may have focussed on accuracy. Instructional methods like Auditory Modelling, Paired Reading, Repeated Reading, and Reader's Theatre could be used (Grabe, 2009). This type of training may help in the development of reading comprehension skills, which is the primary aim of reading instruction. The addition of ORF in remedial training for these learners would also help them to read aloud fluently, enabling them to successfully transition to silent reading, which is the preferred modality of reading in adult life.

This study faced certain limitations owing to the small sample size. Future studies with larger sample groups would help in obtaining 
a clearer understanding of the relationship between ORF and reading comprehension. These studies should also delve into the impacts of the elements of ORF and ORF instructional methods at different levels of comprehension. The potential of this type of research to improve the quality of reading instruction is farreaching and demands exploration.

\section{References}

Alderson, J. C. (2000). Assessing reading. Cambridge, UK: Cambridge University Press.

Argyle, S. (1989). Miscue analysis for classroom use. Reading Horizons. Winter, 93-102.

Carver, R. P. (1997). Reading for one second, one minute, or one year from the perspective of rauding theory. Scientific Studies of Reading, 1, 3-43.

Gibson, E. J. (1965). Learning to read. In H. Singer \& R. B. Ruddell (Eds.) Theoretical models and processes of reading, 252- 259. Newark, Delaware: International Reading Association.

Grabe, W. (2009). Reading in a second language: moving from theory to practice. New York: Cambridge University Press.

Hasbrouck, J. E., \& Tindal, G. (1992). Curriculum-based oral reading fluency norms for students in grades 2 through 5. Teaching Exceptional Children, 24(3), 41-44.

Hudson, R. F., Lane, H. B., \& Pullen, P. C. (2005). Reading fluency assessment and instruction: what, why, and how? The Reading Teacher, 58(8), 702-714.

Kuhn, M., \& Stahl, S. (2003). Fluency: A Review of developmental and remedial practices. Journal of Educational Psychology, 3-21.

Kumaravadivelu, B. (1994). The Postmethod Condition: (E) merging Strategies for Second/Foreign Language Teaching. TESOL Quarterly, 28(1), 27-48.

National Reading Panel. (2000). Teaching children to read: An evidence-based assessment of the scientific research literature on reading and its implications for reading instruction. Washington, DC: National Institute of Child Health and Human Development.

Norris, A. (2012). Ctrl-Z. Retrieved from https:// anorrissbooks.wordpress.com/ books-by-andrew-norriss/ctrl-z-byandrew-norriss/

Norris, A. (2012). The Touchstone. Retrieved from https:// anorrissbooks.wordpress.combooks-by-andrew-norriss/thetouchstone-by-andrew-norriss/ 
Perfetti, C. A. (1999). Comprehending written language: A blueprint of the reader. The Neurocognition of Language, 167-208. doi:10.1093/ acprof:oso/9780198507932.003.0006

Rasinski, T. V. (2004). Assessing reading fluency. Retrieved July 9, 2016, from http:/ / files.eric.ed.gov/fulltext/ED483166.pdf

Rasinski, T., Rikli, A., \& Johnston, S. (2009). Reading fluency: more than automaticity? More than a concern for the primary grades? Literacy Research and Instruction, 48(4), 350-361.

Weir, C., Hawkey, R., Green, A., Unaldi, A., \& Devi, S. (2009). The relationship between the academic reading construct as measured by IELTS and the reading experiences of students in their first year of study at a British university. International English Language Testing System (IELTS) Research Reports 2009, 9, 97. 